Original Article

\title{
Impact of Airflow Limitation on Comorbidities and Postoperative Complications in Patients Undergoing Thoracic Surgery: A Retrospective Observational Study
}

\author{
Kaku Yoshimi, MD, PhD, ${ }^{1}$ Shiaki Oh, MD, PhD,${ }^{2}$ Kenji Suzuki, MD, PhD, ${ }^{2}$ \\ Yuzo Kodama, MD, PhD, ${ }^{1}$ Mitsuaki Sekiya, MD, PhD, ${ }^{1}$ Kuniaki Seyama, MD, PhD,, 3 \\ and Yoshinosuke Fukuchi MD, $\mathrm{PhD}^{1,3}$
}

\begin{abstract}
Purpose: To assess the frequency of airflow limitation (AFL), and the relationship between AFL and preoperative comorbidities or postoperative complications in patients who had undergone thoracic surgery.

Methods: The medical records of patients who underwent non-cardiac thoracic surgery at our institution between August 1996 and January 2013 were retrospectively reviewed. On the basis of preoperative pulmonary function tests, patients were classified with those with $\mathrm{FEV}_{\mathbf{1}} / \mathrm{FVC}<\mathbf{7 0 \%}$ [AFL(+) group] or with $\mathrm{FEV}_{\mathbf{1}} / \mathrm{FVC} \geq 70 \%$ [AFL(-) group]. Patient characteristics, preoperative comorbidities and postoperative complications were compared between the groups.

Results: Of the 3667 patients assessed, $738(20.1 \%)$ were allocated to the AFL $(+)$ group. AFL was an independent risk factor for three preoperative comorbidities: chronic obstructive pulmonary disease (odds ratio [OR]: 4.65), bronchial asthma (OR 4.30) and cardiac diseases (OR 1.41). Airflow limitation was also an independent risk factor for postoperative respiratory failure including long-term oxygen therapy (OR 2.14) and atelectasis (OR 1.90) in the patients who underwent lobectomy or partial resection of the lung.

Conclusions: Our retrospective study revealed that careful attention needs to be paid to airflow limitation in patients who undergo non-cardiac thoracic surgery since it appears to be an important feature of preoperative comorbidities and to increase postoperative complications.
\end{abstract}

Keywords: COPD, comorbidity, respiratory failure, arrhythmia, thoracic surgery

\footnotetext{
${ }^{1}$ Division of Respiratory Medicine, Juntendo University Faculty of Medicine and Graduate School of Medicine, Tokyo, Japan

${ }^{2}$ Division of General Thoracic Surgery, Juntendo University Faculty of Medicine and Graduate School of Medicine, Tokyo, Japan ${ }^{3}$ GOLD Japan Committee, Tokyo, Japan
}

Received: September 30, 2015; Accepted: January 31, 2016 Corresponding author: Kuniaki Seyama, MD, PhD. Division of Respiratory Medicine, Juntendo University Faculty of Medicine and Graduate School of Medicine, 2-1-1 Hongo, Bunkyo-Ku, Tokyo 113-8421, Japan

Email: kseyama@juntendo.ac.jp

(C)2016 The Editorial Committee of Annals of Thoracic and Cardiovascular Surgery. All rights reserved.

\section{Introduction}

Respiratory function tests are one of the routine preoperative assessments of patients undergoing surgery under general anesthesia, and predict postoperative complications and mortality. ${ }^{1,2)}$ It is not unusual to detect unexpected abnormalities of pulmonary function that had not been diagnosed until the tests were undertaken, for example, chronic obstructive pulmonary disease (COPD) or asthma, both of which exhibit obstructive ventilator impairment. COPD is a common disease with a worldwide increase in the number of patients. In an epidemiological study in 
Japan, airflow limitation was detected on respiratory function tests in $10.9 \%$ of adults aged 40 years or older, suggesting that the potential number of patients in Japan with COPD is 5 million or more. ${ }^{3)}$ Consequently, previously undiagnosed airflow limitation is likely to be detected in a substantial proportion of patients when spirometry is performed as preoperative test.

In the retrospective study by Gupta et al., the preoperative comorbidities and postoperative complications of 468795 patients who underwent surgery were examined, and comparisons made between those with and those without COPD. They reported that patients with COPD had higher rates of other comorbidities and significantly higher rates of postoperative complications, including prolonged hospitalization and higher mortality. ${ }^{4)}$ Similar findings have been reported by other investigators, although not yet in Japan. Accordingly, we retrospectively assessed the frequency of airflow limitation, and the relationships between airflow limitation and preoperative comorbidities and postoperative complications, in patients who underwent thoracic surgery (excluding intra-thoracic cardiovascular surgery) under the care of the Department of General Thoracic Surgery, Juntendo University Hospital, Japan.

Here we present our findings that the existence of airflow limitation is not only an important feature of preoperative comorbidities, but also an independent risk factor strongly influencing postoperative outcome after thoracic surgery.

\section{Patients and Methods}

We retrospectively analyzed the medical records of patients who underwent non-cardiac thoracic surgery at our institution between August 1996 and January 2013. The cohort was classified into one of two groups on the basis of the presence of airflow limitation detected during preoperative assessment, and then the characteristics, preoperative comorbidities, and postoperative complications were compared between the groups. We defined airflow limitation as a forced expiratory volume in the first second to forced vital capacity ratio $\left(\mathrm{FEV}_{1} / \mathrm{FVC}\right)<70 \%$ and allocated these patients to the airflow limitation $(\mathrm{AFL})(+)$ group. The remaining patients with $\mathrm{FEV}_{1} / \mathrm{FVC}$ $\geq 70 \%$ were allocated to the AFL(-) group. This retrospective study was approved by the Institutional Review Board of Juntendo University Hospital (IRB No. 26-244). All participants gave us written informed consent for participation in the study.

\section{Statistical analysis}

Data are reported as mean \pm standard deviation (SD). We used the unpaired t-test to compare continuous data whereas the chi-square test or Fisher's exact test for categorical data. To identify the preoperative comorbidities and postoperative complications to which airflow limitation independently contributed, we undertook two step analyses as follows; 1) we used Fisher's exact test to identify comorbidities or complications which show a significant difference between AFL(+) and AFL(-) group, and 2) then we performed multivariate logistic regression analysis using the identified comorbidities or complications as response variable while airflow limitation as the explanatory variable was adjusted by age, sex, history of smoking, and comorbidities or postoperative complications. For each identified comorbidity and complication, odds ratios (ORs) for airflow limitation and $95 \%$ confidence intervals (95\% CIs) were calculated. All analyses were undertaken using SPSS statistics software (version 19, IBM, Tokyo, Japan).

\section{Results}

\section{Characteristics of study population}

The clinical characteristics of the study population are shown in Table 1. The total number of patients assessed was 3667 with a mean age of $61.2 \pm 13.8$ years. The population consisted of 2184 men and 1483 women. An approximately half of the patients $(51.5 \%)$ had a smoking history. On respiratory function testing, mean vital capacity (VC) was $3.72 \pm 2.43 \mathrm{~L}(95.7 \pm 17.7 \%$ predicted), mean $\mathrm{FEV}_{1}$ was $2.34 \pm 1.60 \mathrm{~L}(100.0 \pm 74.9 \%$ predicted $)$, and mean $\mathrm{FEV}_{1} / \mathrm{FVC}$ was $76.5 \pm 9.8 \%$. The most common indication for surgery was primary lung cancer, which was the primary diagnosis in 2072 patients (accounting for $61.5 \%$ of all underlying disease), followed by metastatic lung tumor and mediastinal tumor, each of which accounted for $11.8 \%$.

Of 3667 patients, $738(20.1 \%)$ had airflow limitation and were allocated to the AFL(+) group whereas the remaining 2929 patients $(79.9 \%)$ to the $\mathrm{AFL}(-)$ group. The mean age of the AFL $(+)$ group was significantly higher: $67.4 \pm 10.1$ years compared with $59.6 \pm 14.2$ years in the AFL $(-)$ group $(\mathrm{p}<0.0001)$. A significantly higher proportion of those in the AFL(+) group were current or ex-smokers (75.2\% compared with $45.3 \%$ in the AFL(-) group, $\mathrm{p}<0.0001)$. The number of pack-years smoked was also significantly higher in the AFL(+) group than the AFL(-) group (43.9 \pm 39.5 and $18.0 \pm 28.1$, respectively; $\mathrm{p}<0.0001)$. 
Table 1 Characteristics of the study population

\begin{tabular}{lcccc}
\hline & Total $(\mathrm{n}=3667)$ & AFL $(+)(\mathrm{n}=738)$ & AFL $(-)(\mathrm{n}=2929)$ & P value \\
\hline Age $^{*}$ & $61.2 \pm 13.8$ & $67.4 \pm 10.1$ & $59.6 \pm 14.2$ & \\
Sex (M/F) & $2184 / 1483$ & $609 / 129$ & $1575 / 1354$ & $<0.0001$ \\
Current and ex-smoker, n (\%) & $1890(51.5)$ & $562(76.2)$ & $1328(45.3)$ & $<0.0001$ \\
$\quad$ Pack-year* & $23.2 \pm 32.4$ & $43.9 \pm 39.5$ & $18.0 \pm 28.1$ & $<0.0001$ \\
Pulmonary function & & & & \\
$\quad$ VC (L) & $3.72 \pm 2.43$ & $3.39 \pm 0.83$ & $3.80 \pm 2.73$ & 0.6825 \\
VC \%pred (\%) & $95.7 \pm 17.7$ & $99.8 \pm 18.7$ & $94.7 \pm 17.3$ & $<0.0001$ \\
FEV 1 (L) & $2.34 \pm 1.60$ & $2.00 \pm 0.58$ & $2.44 \pm 1.75$ & $<0.0001$ \\
FEV $_{1} \%$ pred (\%) & $100.0 \pm 74.9$ & $86.1 \pm 19.6$ & $103.5 \pm 82.9$ & $<0.0001$ \\
FEV $_{1}$ /FVC & $76.5 \pm 9.8$ & $62.3 \pm 7.4$ & $80.1 \pm 6.6$ & $<0.0001$ \\
\hline
\end{tabular}

*Data are presented as mean \pm SD. ${ }^{\text {IS }}$ Statistical significance between AFL (+) and AFL (-) groups; chi-square test was utilized for sex and the proportion of current and ex-smokers whereas unpaired t-test for other variables.

VC: vital capacity; $\mathrm{FEV}_{1}$ : forced expiratory volume in 1 second; FVC: forced vital capacity; SD: standard deviation; AFL: airflow limitation

The results of preoperative respiratory function tests showed that mean $\mathrm{FEV}_{1}$ was significantly lower in the $\mathrm{AFL}(+)$ group than the AFL $(-)$ group $(2.00 \pm 0.58 \mathrm{~L}$ compared with $2.44 \pm 1.75 \mathrm{~L}$ in the AFL(-) group, $\mathrm{p}<0.0001$ ), as were mean $\mathrm{FEV}_{1} / \mathrm{FVC}(62.3 \pm 7.4 \%$ compared with $80.1 \pm 6.6 \%$, respectively; $\mathrm{p}<0.0001$ ), and $\mathrm{FEV}_{1} \%$ predicted $(86.1 \pm 19.6 \%$ compared with $103.5 \pm 82.9 \%$, respectively; $\mathrm{p}<0.0001)$. Conversely $\mathrm{VC} \%$ predicted was significantly higher in the AFL(+) group $(99.8 \pm 18.7 \%$ compared with $94.7 \pm 17.3 \%$ in the AFL(-) group, $\mathrm{p}<0.0001$ ).

\section{Preoperative comorbidities}

The prevalence of preoperative comorbidities is presented in Table 2. Notably, only $17.9 \%$ of patients in the AFL(+) group had been diagnosed with COPD before preoperative respiratory function testing. A significantly higher proportion of patients in the AFL (+) group had preoperative diagnoses of hypertension, diabetes mellitus, cardiac diseases, COPD, bronchial asthma, and central nervous system disease. On the other hand, the prevalence of collagen vascular diseases as a preoperative comorbidity was significantly less in AFL(+) group as compare with that in AFL(-) group.

The influence of airflow limitation on preoperative comorbidities was also assessed by means of multivariate logistic regression analysis (Table 3). Airflow limitation remained to be an independent risk factor for cardiac diseases (OR 1.41; 95\% CI 1.10-1.80), COPD (OR 4.65; 95\% CI 3.34-6.51) and bronchial asthma (OR 4.30, 95\% CI 2.60-7.04). Interestingly, the existence of airflow limitation resulted in the lower risk of diabetes mellitus (OR
$0.72,95 \%$ CI $0.54-0.94)$ and collagen vascular diseases (OR $0.45,95 \%$ CI 0.21-0.86).

\section{Postoperative complications}

Next, we analyzed how airflow limitation affected the incidences of postoperative complications. When we categorized operative procedures of thoracic surgeries, they consisted of pneumonectomy $(\mathrm{n}=74)$, lobectomy ( $n=1650)$, partial resection of the lung $(n=1286)$, operation of mediastinal diseases $(n=540)$, and the other miscellaneous procedures $(\mathrm{n}=117)$ such as chest wall resection, lymph node biopsy, operation of trachea, operation of empyema, operation of diaphragm, partial resection of vertebrate, pleural biopsy, sympathetic nerve resection, thoracoplasty, and so on. Since the type of operative procedures is expected to greatly influence the type and incidence of postoperative complications, we focused our analysis on both lobectomy and partial resection of the lung because of the following reasons. First, both procedures have sufficient numbers for statistical analysis. Second, the surgical procedures are neither quite complicated nor too much invasive. Lastly, the categorization itself can generate a relatively homogenous group. In this context, Table 3 shows the comparison of postoperative complications, operation time, bleeding during operation in patients who underwent lobectomy or partial resection of the lung between AFL(+) and AFL(-) groups. A significantly higher proportion of patients in the AFL(+) group had postoperative complication of respiratory failure including long-term oxygen therapy, atelectasis, re-operation, arrhythmias, and refractory air-leakage. In addition, 
Table 2 Comparison of comorbidities between the AFL(+) and AFL(-) groups

\begin{tabular}{lccccc}
\hline & $\begin{array}{c}\text { Total } \\
(\mathrm{n}=3667) \mathrm{n}(\%)\end{array}$ & $\begin{array}{c}\text { AFL }(+) \\
(\mathrm{n}=738) \mathrm{n}(\%)\end{array}$ & $\begin{array}{c}\text { AFL }(-) \\
(\mathrm{n}=2929) \mathrm{n}(\%)\end{array}$ & $\begin{array}{c}\text { Odds ratio for } \\
\text { AFL }(95 \% \text { CI })\end{array}$ & $\begin{array}{c}\text { P value* } \\
\text { Hypertension }\end{array}$ \\
\hline Dyslipidemia & $280(23.2)$ & $210(28.5)$ & $646(22.1)$ & $1.41(1.17-1.70)$ & 0.0003 \\
Diabetes mellitus & $409(11.1)$ & $63(8.5)$ & $217(7.4)$ & $1.16(0.85-1.57)$ & 0.3141 \\
Cardiac diseases & $476(12.9)$ & $154(14.1)$ & $305(10.4)$ & $1.42(1.11-1.81)$ & 0.0048 \\
COPD & $200(5.4)$ & $132(17.9)$ & $319(10.9)$ & $2.22(1.78-2.75)$ & $<0.0001$ \\
Bronchial asthma & $99(2.7)$ & $35(4.7)$ & $68(2.3)$ & $9.17(6.70-12.6)$ & $<0.0001$ \\
Interstitial pneumonia & $192(5.2)$ & $30(4.1)$ & $162(5.5)$ & $2.23(1.42-3.46)$ & $<0.0001$ \\
CNS diseases & $149(4.1)$ & $48(6.5)$ & $101(3.4)$ & $3.45(1.34-2.81)$ & 0.0004 \\
Malignant diseases & $813(22.1)$ & $168(22.8)$ & $645(22.0)$ & $1.04(0.85-1.26)$ & 0.7278 \\
CVD & $110(3.0)$ & $11(1.5)$ & $99(3.4)$ & $0.43(0.21-0.82)$ & 0.0053 \\
Renal diseases & $68(1.8)$ & $13(1.8)$ & $55(1.9)$ & $0.94(0.47-1.75)$ & 1.0000 \\
\hline
\end{tabular}

*Fisher's exact test was utilized to test a statistical significance between AFL(+) and AFL(-) group. Cardiac diseases include previous myocardial infarction, angina pectoris, cardiomyopathy, valvular diseases and aortic aneurysm. Central nervous system diseases include cerebral infarction, transient ischemic attack, Parkinson's disease and other degenerative diseases. AFL: airflow limitation; COPD: chronic obstructive pulmonary disease; CNS: central nervous system; CVD: collagen vascular diseases; $95 \% \mathrm{CI}$ : $95 \%$ confidence interval

Table 3 Comorbidities to which AFL independently contributed

\begin{tabular}{lcr}
\hline Response variable & Odds ratio for AFL $(95 \% \mathrm{CI})$ & P value* $^{*}$ \\
\hline Cardiac diseases & $1.41(1.10-1.80)$ & 0.0057 \\
Diabetes mellitus & $0.72(0.54-0.94)$ & 0.0177 \\
COPD & $4.65(3.34-6.51)$ & $<0.0001$ \\
Bronchial asthma & $4.30(2.60-7.04)$ & $<0.0001$ \\
CVD & $0.45(0.21-0.86)$ & 0.0230 \\
\hline
\end{tabular}

*Multivariate logistic regression analysis was utilized in each response variable (odds ratio for AFL as the explanatory variable was adjusted by age, sex, history of smoking, and comorbidities except for a response variable of interest). AFL: airflow limitation; 95\% COPD: chronic obstructive pulmonary disease; CVD: collagen vascular diseases; CI: $95 \%$ confidence interval

operation time was significantly longer and bleeding during operation was more in $\mathrm{AFL}(+)$ group.

When multivariate logistic regression analysis was performed, a significantly higher proportion of patients in the $\mathrm{AFL}(+)$ group were diagnosed with postoperative respiratory failure that needed supplemental oxygen therapy (OR 2.14, 95\% CI 1.12-4.11) and atelectasis (OR 1.90, 95\% CI 1.19-3.01) (Table 5), indicating that airflow limitation was an independent risk factor for these two types of postoperative complications.

\section{Discussion}

Our findings suggest that airflow limitation is an important feature of preoperative comorbidities and influences postoperative outcome after thoracic surgery. We found that approximately $20 \%$ of patients who underwent thoracic surgery fulfilled the criterion for airflow limitation; and that those affected were significantly more likely to have preoperative comorbidities, such as COPD, bronchial asthma, and cardiac diseases. Furthermore, the incidence of postoperative complications was higher in those with airflow limitation, which proved to be an independent risk factor for postoperative respiratory failure and atelectasis in patients who underwent lobectomy or partial resection of the lung.

A diagnosis of COPD can be established if other causes or conditions resulting in airflow limitation were excluded. In our cohort, only $17.9 \%$ of those with $\mathrm{FEV}_{1} / \mathrm{FVC}<70 \%$ on preoperative respiratory function testing had previously been diagnosed with COPD, strongly suggesting that COPD is under-diagnosed in current clinical practice. This low incidence of the diagnosis of COPD in our study population can be explained by the several reasons; the 
Table 4 Comparison of postoperative complications, operation time and bleeding during operation in patients who underwent lobectomy or partial resection of the lung between AFL(+) and AFL(-) groups

\begin{tabular}{|c|c|c|c|c|c|}
\hline & $\begin{array}{c}\text { Total }(\mathrm{n}=2912) \\
\mathrm{n}(\%)\end{array}$ & $\begin{array}{c}\text { AFL }(+) \\
(\mathrm{n}=623) \mathrm{n}(\%)\end{array}$ & $\begin{array}{c}\text { AFL }(-) \\
(\mathrm{n}=2289) \mathrm{n}(\%)\end{array}$ & $\begin{array}{l}\text { Odds ratio } \\
95 \% \mathrm{CI}\end{array}$ & $\mathrm{P}$ value* \\
\hline $\begin{array}{l}\text { Respiratory failure } \\
\text { including LTOT }\end{array}$ & $48(1.6)$ & $26(4.2)$ & $22(1.0)$ & $4.48(2.42-8.37)$ & $<0.0001$ \\
\hline Atelectasis & $99(3.4)$ & $45(7.2)$ & $54(2.4)$ & $3.22(2.10-4.93)$ & $<0.0001$ \\
\hline Re-operation & $42(1.4)$ & $20(3.2)$ & $22(1.0)$ & $3.42(1.76-6.61)$ & 0.0002 \\
\hline Arrhythmias & $220(7.6)$ & $78(12.5)$ & $142(6.2)$ & $2.16(1.59-2.92)$ & $<0.0001$ \\
\hline Refractory air-leakage & $224(7.7)$ & $79(12.7)$ & $145(6.3)$ & $2.15(1.58-2.89)$ & $<0.0001$ \\
\hline Chylothorax & $59(2.0)$ & $15(2.4)$ & $44(1.9)$ & $1.26(0.65-2.32)$ & 0.4254 \\
\hline Bronchial stump fistula & $8(0.3)$ & $3(0.5)$ & $5(0.2)$ & $2.21(0.34-11.4)$ & 0.3797 \\
\hline Acute exacerbation of IP & $7(0.2)$ & $1(0.2)$ & $6(0.3)$ & $0.61(0.01-5.06)$ & 1.0000 \\
\hline $\begin{array}{l}\text { Wound infection and/or } \\
\text { pyrothorax }\end{array}$ & $17(0.6)$ & $6(1.0)$ & $11(0.5)$ & $2.01(0.61-5.97)$ & 0.2290 \\
\hline Pneumonia & $24(0.8)$ & $7(1.1)$ & $17(0.7)$ & $1.52(0.53-3.87)$ & 0.3250 \\
\hline Operation time (min) & $160.8 \pm 86.3$ & $179.9 \pm 93.0$ & $155.6 \pm 83.6$ & $24.4 \pi(16.2-32.5)$ & $<0.0001$ \\
\hline Bleeding $(\mathrm{ml})$ & $131.4 \pm 317.3$ & $165.4 \pm 354.5$ & $122.1 \pm 305.7$ & $43.3^{\pi}(12.3-74.2)$ & 0.0062 \\
\hline
\end{tabular}

Data of operation time (min) and bleeding $(\mathrm{ml})$ during operation are presented as mean \pm SD. *Fisher's exact test was utilized to test a statistical significance between AFL(+) and AFL(-) groups. Unpaired t-test was utilized for operation time or bleeding during operation to test the difference between AFL(+) and AFL(-) groups. "The values indicate the difference (time or ml) between AFL(+) and AFL(-) groups. AFL: airflow limitation; 95\% CI: 95\% confidence interval; LTOT: long-term oxygen therapy; IP: interstitial pneumonia; SD: standard deviation

Table 5 Postoperative complications in patients who underwent lobectomy or partial resection of the lung to which AFL independently contributed

\begin{tabular}{lcc}
\hline Response variable & Odds ratio for AFL $(95 \% \mathrm{CI})$ & P value* \\
\hline Respiratory failure including LTOT & $2.14(1.12-4.11)$ & 0.0214 \\
Atelectasis & $1.90(1.19-3.01)$ & 0.0063 \\
\hline
\end{tabular}

*Multivariate logistic regression analysis was utilized in each response variable (odds ratio for AFL as the explanatory variable was adjusted by age, sex, history of smoking, and postoperative complications except for a response variable of interest). AFL: airflow limitation; LTOT: long-term oxygen therapy; 95\% CI: 95\% confidence interval

severity of airflow limitation detected was commonly mild, suggesting that they were likely asymptomatic despite fulfilling the diagnostic criterion.

Our findings are similar to those reported by other investigators. It is widely recognized that COPD frequently co-exists with other disease, particularly cardiovascular diseases, such as hypertension and cardiac failure, cerebrovascular disease, osteoporosis and gastrointestinal ulcer disease, all of which have been reported to be significantly more common in patients with COPD presenting for surgery. ${ }^{5-7)}$ Cigarette smoking is a key risk factor for COPD, and is thought to trigger the disease by provoking a systemic inflammatory response; other comorbidities are thought to develop in other organ systems as a consequence of this systemic inflammation. ${ }^{8)}$ The proportion of patients with a history of smoking was significantly higher in the AFL(+) group and, even if they only had mild COPD, smoking seems to have evoked systemic inflammation that consequently provoked a variety of other comorbidities.

The variety and incidence of postoperative complications after pneumonectomy have been examined in a number of studies, all of which reported that acute respiratory failure was the most common, ${ }^{9-11)}$ but, whether preoperative $\mathrm{FEV}_{1}$ and $\mathrm{FEV}_{1} / \mathrm{FVC}$, and predicted postoperative $\mathrm{FEV}_{1}$ are independent risk factors remains unclear. ${ }^{11,12)} \mathrm{In}$ our study, however, we excluded pneumonectomy from the analysis of postoperative complications since the number of pneumonectomy was too small to perform multivariate logistic regression analysis. On the other hand, we found that the preoperative airflow limitation was significantly associated with increased likelihood of postoperative complications after lobectomy or partial resection of the lung: postoperative respiratory failure and atelectasis. Recently, Zhang et al. reported that lung function (postoperative 
$\mathrm{FEV}_{1} \%$ predicted and postoperative diffusing capacity $\%$ predicted) were predictive of pulmonary complications after both open and minimally invasive lobectomy. ${ }^{13)} \mathrm{Sim}$ ilarly, the other studies indicated that COPD is the preoperative variable increasing postoperative atelectasis after surgery for non-small cell lung cancer ${ }^{12)}$ and for lobectomy. ${ }^{14,15)}$ Stolz et al. reported that patients with COPD and those undergoing right upper lobectomy are at higher risk of postlobectomy atelectasis. ${ }^{14)}$ In our study, regardless of the existence of underlying diseases, the presence of airflow limitation itself was an independent risk factor for postoperative respiratory failure and atelectasis, which appears to be in broad agreement with these reports.

Large-scale epidemiological studies of COPD, including those conducted in Japan (the NICE study) and internationally (the BOLD and PLATINO studies) have reported that the prevalence of COPD is higher than previously thought. ${ }^{3,16-18)}$ The results of these epidemiological studies are greatly influenced by the methods used for surveillance and patient selection, and diagnostic criteria for COPD, nonetheless COPD still seems to be underdiagnosed worldwide. This is a likely cause that we happen to identify airflow limitation in a preoperative respiratory function test, and eventually establish a diagnosis of COPD in patients who are about to have a surgery due to a certain disease. As found in this study, pre-operative patients with airflow limitation have higher rates of non-respiratory comorbidities such as cardiac diseases and thus a comprehensive approach should be considered so that diseases of other major organ systems are not overlooked. Furthermore, it needs to realize that another inflammatory airway disease, bronchial asthma, is likely to exist.

A limitation of our study is that it was retrospective and observational. We relied on the integrity of data recorded in our clinical databases, and comorbid diseases such as COPD and bronchial asthma may not have been recorded accurately in some cases. Furthermore, diagnoses of postoperative complications were based on the clinical judgment of individual physicians in our department, so may have been influenced by inter-individual variation. The significance of impact of airflow limitation on postoperative complications and comorbidities will need to be clarified by future studies that collect data prospectively, and also include patients undergoing abdominal surgery, to allow a fuller understanding of the impact of airflow limitation on the outcomes of patients undergoing a broader range of major surgical procedures to be obtained.

\section{Conclusion}

Both physicians and surgeons in pulmonary medicine should aware the significance of airflow limitation which predicts unperceived existence of preoperative comorbidities as well as an independent risk strongly influencing postoperative outcome.

\section{Acknowledgements}

The authors wish to acknowledge the editorial assistance from GOLD Japan committee.

\section{Disclosure Statement}

The authors have nothing to disclose with regard to commercial support.

\section{References}

1) American College of Physicians. Preoperative pulmonary function testing. Ann Intern Med 1990; 112: 793-4.

2) Gass GD, Olsen GN. Preoperative pulmonary function testing to predict postoperative morbidity and mortality. Chest 1986; 89: 127-35.

3) Fukuchi Y, Nishimura M, Ichinose M, et al. COPD in Japan: the Nippon COPD Epidemiology study. Respirology 2004; 9: 458-65.

4) Gupta H, Ramanan B, Gupta PK, et al. Impact of COPD on postoperative outcomes: results from a national database. Chest 2013; 143: 1599-606.

5) Miller J, Edwards LD, Agustí A, et al. Comorbidity, systemic inflammation and outcomes in the ECLIPSE cohort. Respir Med 2013; 107: 1376-84.

6) Clarenbach CF, Thurnheer R, Kohler M. Vascular dysfunction in chronic obstructive pulmonary disease: current evidence and perspectives. Expert Rev Respir Med 2012; 6: 37-43.

7) Lahousse L, Vernooij MW, Darweesh SKL, et al. Chronic obstructive pulmonary disease and cerebral microbleeds: the Rotterdam study. Am J Respir Crit Care Med 2013; 188: 783-8.

8) Fabbri LM, Luppi F, Beghé B, et al. Complex chronic comorbidities of COPD. Eur Respir J 2008; 31: 204-12.

9) Hubaut JJ, Baron O, Al Habash O, et al. Closure of the bronchial stump by manual suture and incidence of bronchopleural fistula in a series of 209 pneumonectomies for lung cancer. Eur J Cardiothorac Surg 1999; 16: 418-23.

10) Bernard A, Deschamps C, Allen MS, et al. Pneumonectomy for malignant disease: factors affecting early morbidity and mortality. J Thorac Cardiovasc Surg 2001; 121: $1076-82$. 
11) Algar FJ, Alvarez A, Salvatierra A, et al. Predicting pulmonary complications after pneumonectomy for lung cancer. Eur J Cardiothorac Surg 2003; 23: 201-8.

12) Sekine Y, Behnia M, Fujisawa T. Impact of COPD on pulmonary complications and on long-term survival of patients undergoing surgery for NSCLC. Lung Cancer 2002; 37: 95-101.

13) Zhang R, Lee SM, Wigfield C, et al. Lung function predicts pulmonary complications regardless of the surgical approach. Ann Thorac Surg 2015; 99: 1761-7.

14) Stolz AJ, Schutzner J, Lischke R, et al. Predictors of atelectasis after pulmonary lobectomy. Surg Today 2008; 38: 987-92.
15) Agostini P, Cieslik H, Rathinam S, et al. Postoperative pulmonary complications following thoracic surgery: are there any modifiable risk factors? Thorax 2010; 65: 815-8.

16) Halbert RJ, Natoli JL, Gano A, et al. Global burden of COPD: systematic review and meta-analysis. Eur Respir J 2006; 28: 523-32.

17) Buist AS, McBurnie MA, Vollmer WM, et al. International variation in the prevalence of COPD (the BOLD Study): a population-based prevalence study. Lancet 2007; 370: 741-50.

18) Menezes AM, Perez-Padilla R, Jardim JR, et al. Chronic obstructive pulmonary disease in five Latin American cities (the PLATINO study): a prevalence study. Lancet 2005; 366: 1875-81. 